\title{
A Multiple Access Technique for Differential Chaos Shift Keying
}

\author{
F.C.M. Lau, M.M. Yip, C.K. Tse and S.F. Hau \\ Department of Electronic and Information Engineering, Hong Kong Polytechnic University, Kowloon, \\ Hong Kong.
}

Tel: (852) 2766-6206 Fax: (852) 2362-8439 Email: encmlau@ polyu.edu.hk

\begin{abstract}
Various chaos-based digital communications techniques have been proposed recently. Among them, differential chaos shift keying (DCSK) allows the receiving end to decode the signal using noncoherent detection. This paper proposes and analyses a multiple access scheme for DCSK. A simple 1-dimensional iterative map has been used to generate the chaotic signals for all users. Bit error probabilities have been derived numerically for different number of users and computer simulations have been performed to verify the results.
\end{abstract}

\section{Introduction}

Chaotic signals are characterised by their sensitive dependence on initial conditions as well as random-like behaviour. Moreover, their continuous broadband power spectrum feature renders them useful in encoding information in communications. The basic chaos shift keying (CSK) [1],[2] maps different symbols to different chaotic attractors, which are produced by a dynamical system for different values of a bifurcation parameter or by completely different dynamical systems. A coherent correlation CSK receiver is then required at the receiving end to decode the signals. Noncoherent detection is also possible provided the signals generated by the different attractors have different attributes, such as mean of the absolute value, variance and standard deviation. However, the optimal decision level of the threshold detector will depend on the signal-to-noise ratio. To overcome the threshold level shift problem, differential CSK (DCSK) is proposed [1],[2]. The advantage of DCSK over CSK is that the threshold level is always set at zero and is independent of the noise effect.

Since CSK/DCSK spreads the spectrum of the data signal over a much larger bandwidth, multiple access becomes an essential feature for practical implementation of the system. Furthermore, it is imperative that more users are included in the same bandwidth without causing excessive interference to one another. In [3], a two-user DCSK system was first proposed. In this paper, a generalized multiple access technique for use with DCSK (MADCSK) is proposed and analysed. The proposed scheme is simple and is in theory scalable to any number of users, provided the low-correlation property is maintained among the chaotic signal segments representing the different users.

\section{System Model and Multiple Access Technique}

In DCSK, every transmitted symbol is represented by two chaotic signal samples. The first one serves as the reference (reference sample) while the second one carries the data (data sample) [1],[2]. If a " $+1 "$ is to be transmitted, the data sample will be identical to the reference sample, and if a " -1 " is to be transmitted, an inverted version of the reference sample will be used as the data sample. In a single-user DCSK system, the reference sample will be transmitted during the first half-bit period and the data sample on the second half-bit period. In a multiple access system, to avoid excessive interference and hence mis-detection, the separation between the reference and data samples must be different for different users.

Suppose there are $N$ users within the system. The chaotic signals $x_{i, k}(i=$ $1,2, \ldots, N)$ of the users are generated by the same map $x_{i, k+1}=g\left(x_{i, k}\right)$. On the other hand, all users are assigned different initial conditions such that different chaotic samples are generated. Assume the system starts at $t=0$ and the binary data to be transmitted has a period of $T_{b}$. Let $2 \alpha$ be the spreading factor, defined as $T_{b} / T_{c}$, where $\alpha$ is an integer. Here we propose a multiple access scheme where the separation between the reference and data samples differs for different users, as illustrated in Fig. 1. For user $i$, each data frame will consist of $2 i$ half-bit slots. The first $i$ half-bit slots in each frame (slots 1 to $i$ ) will be used to transmit the $i$ reference samples while the remaining $i$ half-bit slots (slots $i+1$ to $2 i$ ) are used to transmit the data samples. If a " +1 " is to be transmitted in slot $i+1$, the sample in slot 1 is repeated in slot $i+1$, otherwise, an inverted copy is sent. Similarly, in slot $i+2$, the same or inverted copy of the sample in slot 2 is sent, and so on. As a result, the reference and data samples of user $i$ will be separated by $i$ half-bit periods. Fig. 2 shows a typical transmitted waveform for user 3 .

Let $y_{i, m, f}(t)$ be the reference sample at the $m$ th half-bit slot $(1 \leq m \leq i)$ of the $f$ th data frame for the $i$ th user, i.e., the reference sample at the [2i(f$1)+m]$ th half-bit slot for the $i$ th user. Thus we have

$$
\begin{aligned}
& y_{i, m, f}(t) \\
& =\sum_{k=0}^{\alpha-1} x_{i, k+\alpha[(m-1)+(f-1) i]} r\left[t-\left(k T_{c}+(m-1) \frac{T_{b}}{2}+(f-1) i T_{b}\right)\right]
\end{aligned}
$$

where $r(t)$ is a rectangular pulse of unit amplitude and width $T_{c}$, i.e.,

$$
r(t)= \begin{cases}1 & 0 \leq t<T_{c} \\ 0 & \text { elsewhere }\end{cases}
$$

From (1) we can write the reference samples of user $i$ as

$$
\operatorname{ref}_{i}(t)=\sum_{f=1}^{\infty} \sum_{m=1}^{i} y_{i, m, f}(t)
$$

Denoting the transmitted data of user $i$ by $\left\{d_{i, 1}, d_{i, 2}, d_{i, 3}, \ldots\right\} \in\{-1,+1\}$, the corresponding data samples are given by

$\operatorname{data}_{i}(t)=\sum_{f=1}^{\infty} \sum_{m=1}^{i} d_{i, m+(f-1) i} y_{i, m, f}\left(t-\frac{i T_{b}}{2}\right)$

and $d_{i, m+(f-1) i} y_{i, m, f}\left(t-\frac{i T_{b}}{2}\right)$ is the transmitted data sample during the $[2 i(f-1)+i+m]$ th half-bit slot. Combining the reference and data samples, we obtain the transmitted waveform of the $i$ th user as

$$
\begin{aligned}
s_{i}(t) & =\operatorname{ref}_{i}(t)+\operatorname{data}_{i}(t) \\
& =\sum_{f=1}^{\infty} \sum_{m=1}^{i}\left[y_{i, m, f}(t)+d_{i, m+(f-1) i} y_{i, m, f}\left(t-\frac{i T_{b}}{2}\right)\right]
\end{aligned}
$$

The overall transmitted signal of the whole system is derived by summing the signals of all individual users, i.e.,

$$
s(t)=\sum_{i=1}^{N} s_{i}(t)
$$

Fig. 3 shows the receiver structure of user $i$. At the receiving end, the half-bit slots in the first half of each frame will correlate with those in the second half. During the same time, the correlator output is sampled every $T_{b} / 2$ before the correlator is reset. The output is then compared with the threshold zero to determine whether a " +1 " or " -1 " has been received. Fig. 4 depicts the correlator output and the decoded symbols of user 3 in a 5-user system, assuming a spreading factor of 2000 . If the correlation between different samples from the same user or samples from different users is low, a low bit error probability (BEP) is expected. 


\section{Numerical Analysis}

As derived in the previous section, the overall transmitted signal is given by $s(t)$. Ignoring the effect of noise and filters, the same signal will arrive at the receiver input. Consider the $m_{t}$ th half-bit slot in the $f_{t}$ th data frame of the $i$ th user, i.e., the $\left[2\left(f_{i}-1\right) i+m_{i}\right]$ th half-bit, where $1 \leq m_{i} \leq i$. At the receiving end, the signal in this half-bit slot will correlate with that in the $\left[2\left(f_{i}-1\right) i+i+m_{i}\right]$ th half-bit slot. The output of the correlator is given by

$O_{i}=\sum_{u=1}^{N} \sum_{v=1}^{N} \int_{\left[2\left(f_{i}-1\right) i+i+m_{i}-1\right] T_{b} / 2}^{\left[2\left(f_{i}-1\right) i+i+m_{i}\right] T_{b} / 2} s_{u}(t) s_{v}\left(t-\frac{i T_{b}}{2}\right) d t$

where $s_{u}(t)$ and $s_{v}(t)$ denote the signals of users $u$ and $v$. For brevity, we define $X_{i, u, v}$ as

$$
X_{i, u, v}=\int_{\left[2\left(f_{i}-1\right) i+i+m_{i}-1\right] T_{b} / 2}^{\left[2\left(f_{i}-1\right) i+i+m_{i}\right] T_{b} / 2} s_{u}(t) s_{v}\left(t-\frac{i T_{b}}{2}\right) d t .
$$

Hence, $O_{i}$ can be written as

$$
O_{i}=\sum_{u=1}^{N} \sum_{v=1}^{N} X_{i, u, v}
$$

In the sequel, we assume that the map $x_{i, k+1}=g\left(x_{i, k}\right)=4 x_{i, k}^{3}-3 x_{i, k} \quad(i=1,2, . ., N)$ is used to generate the chaotic coefficients for user $i$. It can be readily shown that with this choice of $g($.$) , the X_{i, u, v}$ 's are all normal random variables [4]. The means and variances of the variables are tabulated in Table 1 for spreading factors 200 and 2000. Therefore, assuming that the $X_{i, u, v}$ 's are also independent, $O_{i}$ is also normal with mean

$\overline{O_{i}}=\left\{\begin{array}{cc}0.5 T_{b} & "+1 " \text { is transmitted } \\ -0.5 T_{b} & "-1 " \text { is transmitted }\end{array}\right.$

and variance

$$
\begin{aligned}
\sigma_{O_{i}}^{2} & =\sigma_{X_{, i, i, i}}^{2}+\sum_{\substack{u=1 \\
u \neq i}}^{N} \sigma_{X_{i, u, u}}^{2}+\sum_{\substack{u=1 v=1 \\
u \neq v}}^{N} \sum_{X_{i, u, v}}^{N} \sigma^{2} \\
& =\sigma_{X_{, i, i, i}}^{2}+\left(N^{2}-1\right) \sigma_{X_{i, u, u}}^{2} .
\end{aligned}
$$

Since the output of the correlator is normally distributed with mean $\overline{O_{i}}$ and variance $\sigma_{O_{i}}^{2}$, the BEP is given by

$$
\mathrm{BEP}=Q\left(\frac{0.5 T_{b}}{\sigma_{O_{i}}}\right)
$$

where the $Q$-function in (12) is defined as

$$
Q(x)=\int_{x}^{\infty} \frac{1}{\sqrt{2 \pi}} \exp \left(\frac{-t^{2}}{2}\right) d t \text {. }
$$

It can be observed from equation (11) that $\sigma_{O_{i}}^{2}$ increases with $N^{2}$. Hence, the performance of the system will degrade quite rapidly with the number of users.

\section{Simulations and Results on Error Performance}

Simulations have been carried out to confirm the feasibility of the proposed multiple access scheme and to verify the foregoing numerical analysis. Spreading factors 200 and 2000 are used and the bit duration $T_{b}$ is taken as $10^{-4} \mathrm{~s}$. The number of users in the system is assigned up to 50 and different initial conditions are assigned to different users to generate the chaotic signals. 10,000 bits are first sent from each user. Then, the number of errors received by each user and the average number of errors among all users are noted. Table 2 compares the numerical BEPs with the simulation results. It can be observed that the simulation results match very closely with the numerical ones. As mentioned in the previous section, $\sigma_{O_{i}}^{2}$ increases with $N^{2}$. Hence, the BEP becomes high when the number of users is large. On the other hand, by using a higher spreading factor and hence lower autocorrelation and cross-corrrelation values, the system performance can be drastically improved.

\section{Conclusions}

In this paper, we have proposed a simple multiple access scheme for use with differential chaos shift keying (MA-DCSK). The access scheme of different users is described and the corresponding noncoherent receiver is also designed to decode the signals. The scheme can theoretically be scaled to any number of users, provided the low-correlation property is maintained among the chaotic signal samples representing the different users. As would be expected, the proposed scheme achieves unbiased error probabilities for all users and the error performance degrades as the number of users increases. However, the spreading factor can be increased to improve performance.

In order to evaluate the performance of the system, a simple 1dimensional iterative map has been used to generate the chaotic signals for all $N$ users. For this particular choice of chaotic generators, it is found that the correlator output follows a normal distribution with variance increases with $N^{2}$. As a consequence, the numerical bit error probability (BEP) of the system is derived. Simulations are then carried out and the results match very closely with the numerical BEP. It is observed that by using chaos generators with a higher spreading factor and hence lower autocorrelation and cross-corrrelation values, the BEP can be reduced.

\section{Acknowledgement}

This work was supported in part by a research grant provided by the Research Committee of Hong Kong Polytechnic University, Hong Kong SAR.

\section{References}

[1] M.P. Kennedy, "Chaotic communications: from chaotic synchronization to FM-DCSK," Proceedings, 6th International Specialist Workshop on Nonlinear Dynamics of Electronics Systems (NDES 98), Budapest, Hungary, pp. 31-40, July 1998.

[2] G. Kolumban, M.P. Kennedy and L.O. Chua, "The role of synchronization in digital communications using chaos - Part II: Chaotic modulation and chaotic synchronization," IEEE Trans. on Circuits and Systems I, Vol. 45, No. 11, pp. 1129-1140, Nov. 1998.

[3] G. Kolumban, M.P. Kennedy and G. Kis, "Multilevel differential chaos keying," Proceedings, 5th International Specialist Workshop on Nonlinear Dynamics of Electronics Systems, Moscow, Russia, June 1997, pp. 191-196.

[4] M.M. Yip, Study of Chaos-based Digital Communications, M.Phil. Thesis, The Hong Kong Polytechnic University, 2000. 


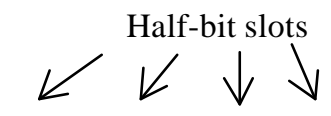

User 1

$$
\begin{aligned}
& \begin{array}{|l|l|l|l|l|l|llllllll}
\hline \mathrm{R}_{1,1} & \mathrm{D}_{1,1} & \mathrm{R}_{1,2} & \mathrm{D}_{1,2} & \mathrm{R}_{1,3} & \mathrm{D}_{1,3} \\
\hline
\end{array} \\
& \begin{array}{|l|l|l|}
\hline \mathrm{F}_{1,1} & \mathrm{~F}_{1,2} & \mathrm{~F}_{1,3} \\
\hline
\end{array}
\end{aligned}
$$

\begin{tabular}{|c|c|c|c|c|c|c|c|}
\hline $\mathrm{R}_{2,1}$ & $\mathrm{R}_{2,2}$ & $\mathrm{D}_{2,1}$ & $\mathrm{D}_{2,2}$ & $\mathrm{R}_{2,3}$ & $\mathrm{R}_{2,4}$ & $\mathrm{D}_{2,3}$ & $\mathrm{D}_{2,4}$ \\
\hline \multicolumn{4}{|c|}{$\mathrm{F}_{2,1}$} & \multicolumn{4}{|c|}{$\mathrm{F}_{2,2}$} \\
\hline
\end{tabular}

User 2

User $N$

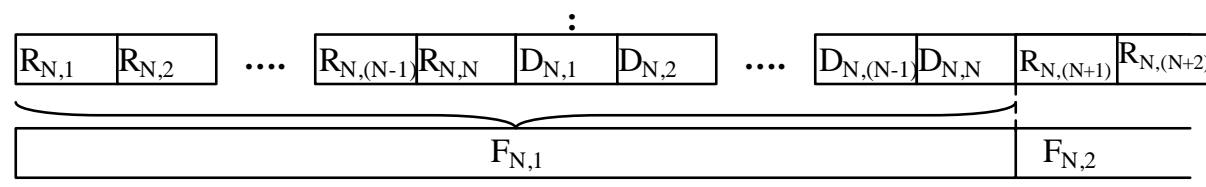

$\mathrm{R}_{\mathrm{i}, \mathrm{j}} \quad j$ th chaos reference sample for user $i \quad \mathrm{D}_{\mathrm{i}, \mathrm{j}} \quad j$ th chaos data sample for user $i$

$\mathrm{F}_{\mathrm{i}, \mathrm{j}} \quad j$ th data frame for user $i$

Figure 1 Transmission scheme in a multiple access differential chaos shift keying (MA-DCSK) system

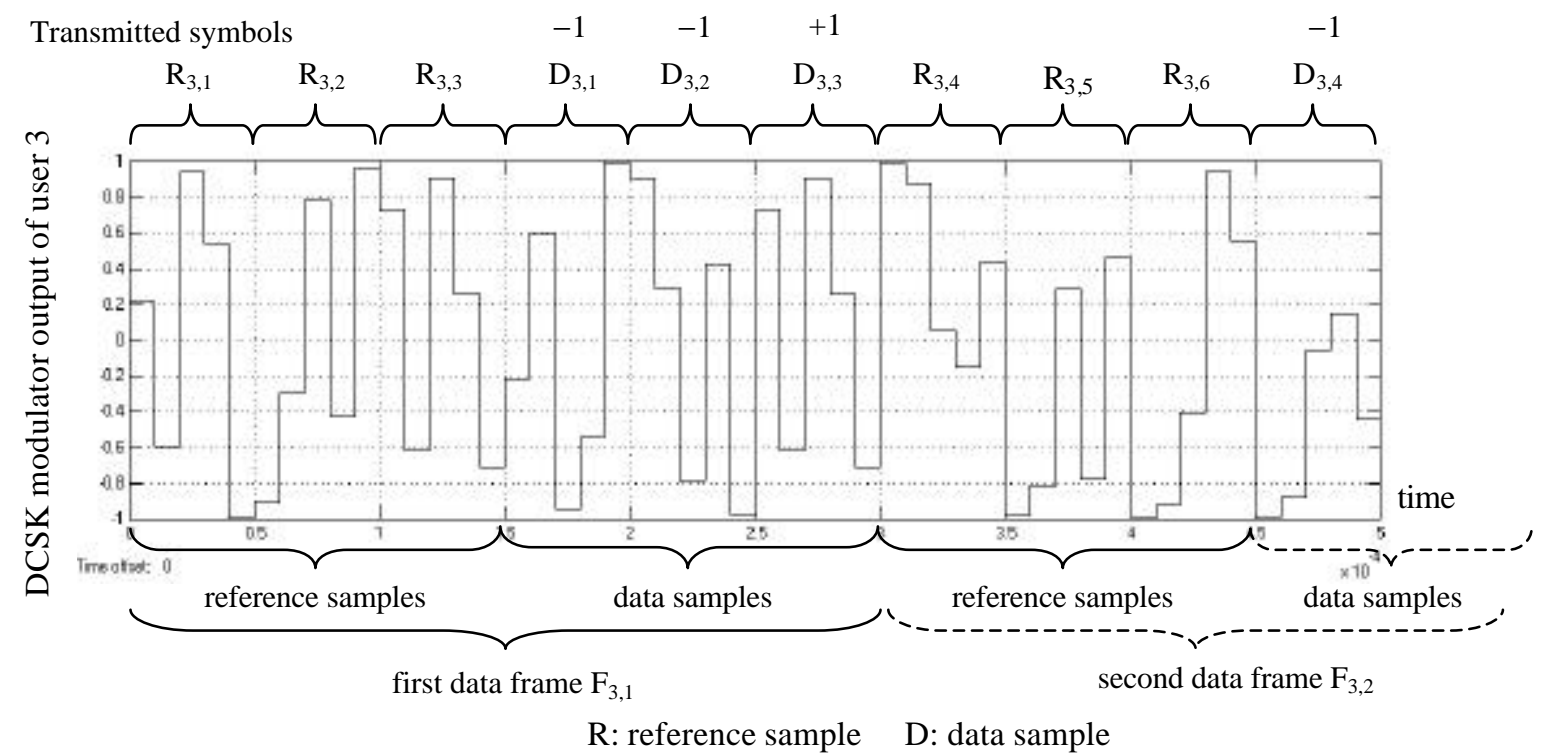

Figure 2 A typical transmitted signal for user 3 in a multiple access DCSK system (spreading factor = 10)

Integrator resets itself every $T_{b} / 2$

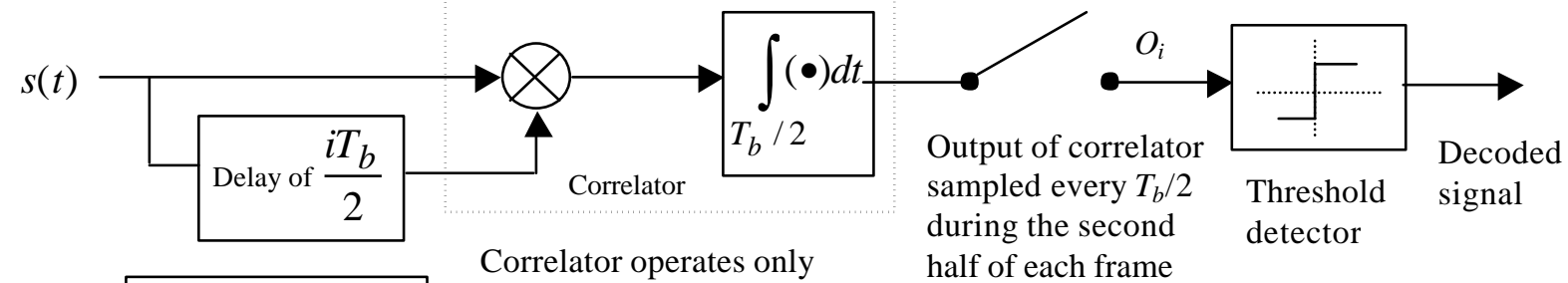

Receiver of user $i$ during the second half of each frame

Figure 3 Multi-user DCSK receiver 


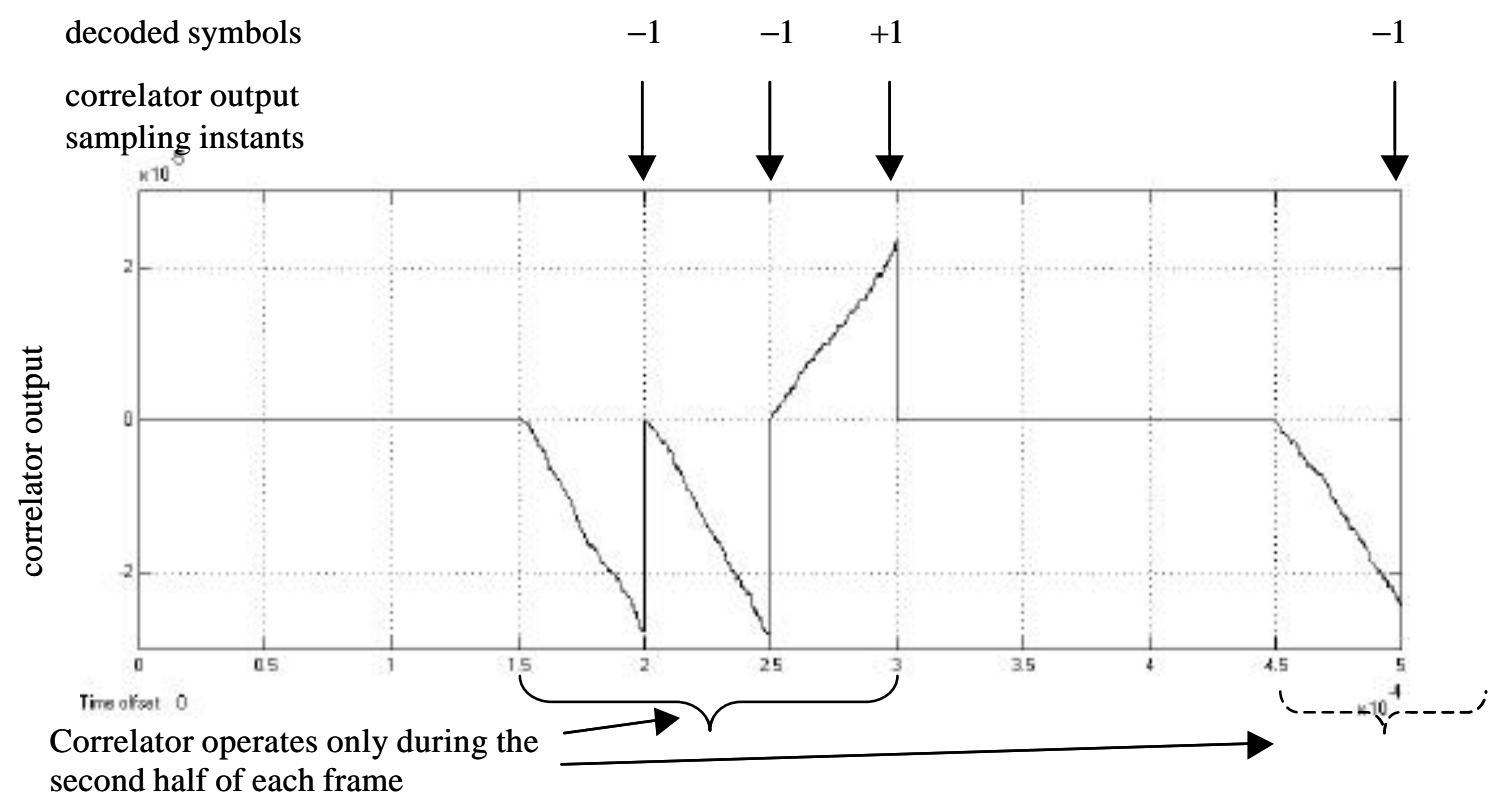

Fig. 4 Output of the correlator and the decoded symbols of user 3 in a 5-user system (spreading factor =2000)

\begin{tabular}{|c|c|c|c|c|}
\hline $\begin{array}{c}\text { Spreading } \\
\text { factor }\end{array}$ & $\begin{array}{c}\text { Statistical } \\
\text { properties }\end{array}$ & Case I: $u=v=i$ & Case II: $u=v, u \neq i$ & Case III: $u \neq v$ \\
\hline 200 & Mean & $\overline{X_{i, i, i}}=\left\{\begin{array}{cc}0.25 T_{b} & \text { "+1" is transmited } \\
-0.25 T_{b} & \text { "- } 1 " \text { is transmited }\end{array}\right.$ & $\overline{X_{i, u, u}}=0$ & $\overline{X_{i, u, v}}=0$ \\
\cline { 2 - 5 } & Variance & $\sigma_{X_{i, i, i}}^{2}=0.31 \times 10^{-3} T_{b}^{2}$ & $\sigma_{X_{i, u, u}}^{2}=0.625 \times 10^{-3} T_{b}^{2}$ & $\sigma_{X_{i, u, v}}^{2}=0.625 \times 10^{-3} T_{b}^{2}$ \\
\hline 2000 & Mean & $\overline{X_{i, i, i}}=\left\{\begin{array}{cc}0.25 T_{b} & \text { "+1" is transmited } \\
-0.25 T_{b} & \text { "- } 1 " \text { is transmited }\end{array}\right.$ & $\overline{X_{i, u, u}}=0$ & $\overline{X_{i, u, v}}=0$ \\
\cline { 2 - 5 } & Variance & $\sigma_{X_{i, i, i}}^{2}=0.31 \times 10^{-4} T_{b}^{2}$ & $\sigma_{X_{i, u, u}}^{2}=0.625 \times 10^{-4} T_{b}^{2}$ & $\sigma_{X_{i, u, v}}^{2}=0.625 \times 10^{-4} T_{b}^{2}$ \\
\hline
\end{tabular}

Table 1 Statistical properties of $X_{i, u, v}$ for spreading factors 200 and 2000

\begin{tabular}{|c|c|c|c|c|}
\hline & \multicolumn{2}{|c|}{ Spreading factor $=200$} & \multicolumn{2}{|c|}{ Spreading factor $=2000$} \\
\hline $\begin{array}{c}\text { No. of users } \\
N\end{array}$ & $\begin{array}{l}\text { Average BEP among all } \\
\text { users by simulation }\end{array}$ & $\begin{array}{l}\text { BEP by numerical } \\
\text { calculation }\end{array}$ & $\begin{array}{c}\text { Average BEP among all } \\
\text { users by simulation }\end{array}$ & $\begin{array}{l}\text { BEP by numerical } \\
\text { calculation }\end{array}$ \\
\hline 1 & 0.0000 & 0.0000 & 0.0000 & 0.0000 \\
\hline 2 & 0.0000 & 0.0000 & 0.0000 & 0.0000 \\
\hline 3 & 0.0001 & 0.0003 & 0.0000 & 0.0000 \\
\hline 4 & 0.0043 & 0.0055 & 0.0000 & 0.0000 \\
\hline 5 & 0.0190 & 0.0217 & 0.0000 & 0.0000 \\
\hline 10 & 0.1562 & 0.1580 & 0.0007 & 0.0008 \\
\hline 15 & 0.2501 & 0.2523 & 0.0166 & 0.0174 \\
\hline 20 & 0.3090 & 0.3084 & 0.0560 & 0.0568 \\
\hline 25 & 0.3453 & 0.3445 & 0.1031 & 0.1029 \\
\hline 30 & 0.3694 & 0.3694 & 0.1453 & 0.1459 \\
\hline 35 & 0.3878 & 0.3875 & 0.1825 & 0.1831 \\
\hline 40 & 0.4022 & 0.4013 & 0.2152 & 0.2146 \\
\hline 45 & 0.4125 & 0.4121 & 0.2418 & 0.2411 \\
\hline 50 & 0.4216 & 0.4207 & 0.2653 & 0.2635 \\
\hline
\end{tabular}

Table 2 Comparison of BEPs from numerical calculation and by simulation 